\title{
2016 Oswald Veblen Prize in Geometry
}

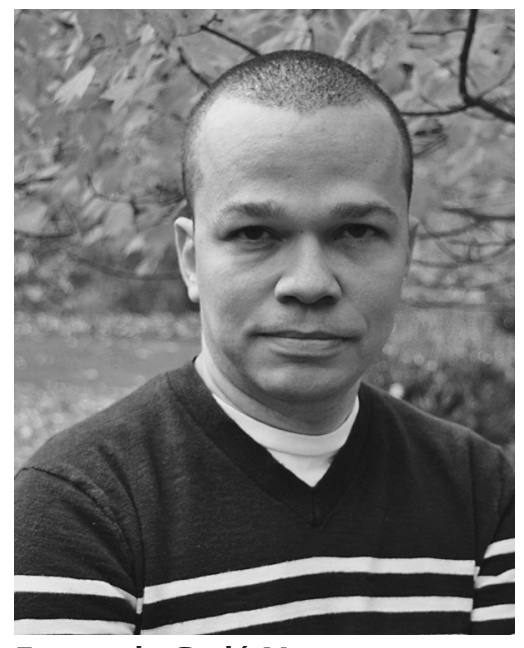

Fernando Codá-Marques

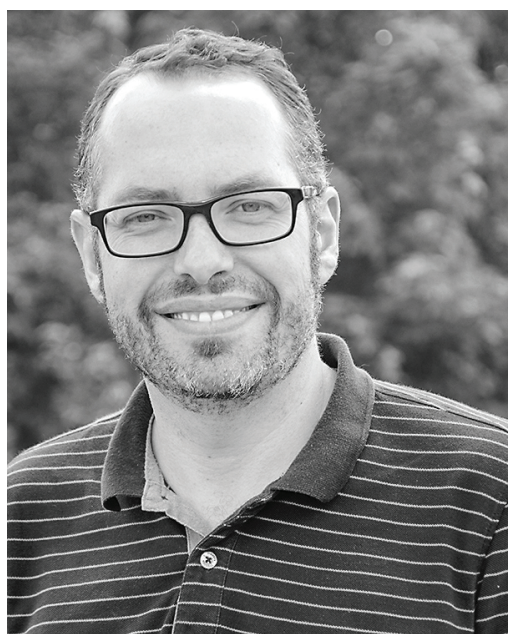

André Neves
FERNANDO CODÁ-MARQUES and ANDRÉ NEVES were awarded the 2016 Oswald Veblen Prize in Geometry at the 122nd Annual Meeting of the AMS in Seattle, Washington, in January 2016.

\section{Citation}

The Willmore conjecture, formulated in 1965, concerns the optimal shape of a torus $T$ smoothly embedded in $\mathbb{R}^{3}$ or $S^{3}$. It states that the Willmore energy of the surface, defined in terms of its mean curvature $H$ by $W(T)=\int_{T} H^{2} d A$, is minimized by the standard Clifford torus. (This torus is defined by $|z|=|w|$ on the unit sphere $S^{3} \subset \mathbb{C}^{2}$.) The energy $W(T)$ is conformally invariant, so images of the standard torus under Möbius transformations should also be minimizers. The study of $W(T)$ dates from the work of Wilhelm Blaschke; physically, it measures the bending energy stored in $T$.

Codá-Marques and Neves proved both the minimizing property of the Clifford torus and its essential uniqueness by studying the minimizer within a five-dimensional space of cycles, building on earlier work of Francisco Urbano (1990) and Antonio Ros (1999).

Their contributions also include two sequels to this work of a similar spirit. The first is their

For permission to reprint this article, please contact: reprint-permission@ams .org.

DOI: http://dx.doi.org/10.1090/noti1358 proof, jointly with Ian Agol, of a conjecture of Michael Freedman, Zheng-Xu He, and Zhenghan Wang (1994) on the energy of links in $S^{3}$. They proved that the Möbius energy $M(L)$ of a nontrivial link $L \subset S^{3}$-another conformal invariant-is minimized by the standard Hopf link, made up of two closed geodesics with linking number one.

The second is their proof of the existence of infinitely many minimal hypersurfaces in a closed manifold of positive Ricci curvature, confirming a conjecture of Shing-Tung Yau (1982). This line of research can be regarded as a deep, higher-dimensional extension of the study of closed geodesics for an arbitrary Riemannian metric on $S^{2}$, dating from the contributions of Lazar' Lyusternik and Lev Schnirel'man in the 1940s. The key difficulty in both cases comes from multiple coverings of the same hypersurface.

Codá-Marques and Neves overcome this fundamental difficulty by using recent results of Misha Gromov and Larry Guth in an ingenious way. In each dimension $p$, the $p$-dimensional topology of the space of codimension- 1 cycles is captured by the notion of a " $p$-sweepout," and Gromov and Guth showed that the minimax level over $p$-sweepouts grows sublinearly with $p$. Combining this result with a well-known theorem of Theodore Frankel-that in positive Ricci curvature minimal hypersurfaces intersect-they were able to rule out 
the possibility that there are only finitely many minimal hypersurfaces.

These sequels are detailed in their papers "Minmax theory and the energy of links" (joint with Agol) and "Existence of infinitely many minimal hypersurfaces in positive Ricci curvature".

The work of Fernando Codá-Marques and André Neves represents a landmark achievement in the use of variational methods in differential geometry.

\section{Biographical Sketch of Codá-Marques}

Fernando Codá-Marques was born in São Carlos in 1979 and grew up in Maceió, state of Alagoas, Brazil. He received a BS from the Federal University of Alagoas (UFAL) and an MS from IMPA in 1999. He received his $\mathrm{PhD}$ from Cornell University in 2003 under the supervision of José Fernando Escobar. He became a professor at Princeton University in 2014, after being at IMPA since 2003. He has visited Stanford University during several periods since 2005, was a member of the Institute for Advanced Study in Princeton (2008), and a visiting professor at several institutions in France, including the École Normale Superieure, Ecole Polytechnique, Institut Henri Poincaré, Institut Fourier, and Marne-laVallée. In 2012 he received the Ramanujan Prize (ICTP, Abel Fund, and IMU), the TWAS Prize in Mathematics, and the UMALCA (Union Matematica de America Latina y el Caribe) Prize.

Codá-Marques gave an invited address at the International Congress of Mathematicians (ICM) in Hyderabad in 2010 and a plenary lecture at the ICM in Seoul in 2014. He also delivered the Barrett Memorial Lectures (University of Tennessee), the Yamabe Lectures (Northwestern), the Hadamard Lectures (IHP-Paris), the S. Lojasiewicz Lecture (Krakow), and the Joseph Fels Ritt Lectures (Columbia University). He is a member of the Brazilian Academy of Sciences since 2014. He lives with his wife, Ana, and his son, Pedro.

\section{Response from Fernando Codá-Marques}

It is an honor and an immense pleasure to be a recipient, together with my friend André, of the prestigious Oswald Veblen Prize in Geometry.

I am thankful to the committee for this recognition of our work. I am grateful to my family, especially my parents, Severino and Dilze, my wife Ana, and my siblings Gustavo and Clarissa. I am sure that without their love and support I would not be here today. I also look forward to meeting my baby son, Pedro, who is joining us.

I thank also my late advisor, José Fernando Escobar (Chepe), who was always kind and supportive of me, and Richard Schoen, whose influence has been fundamental in my career. The year I spent with Rick was decisive and helped shape my vision of what is important in mathematics. I thank all my teachers, especially Professor Manfredo do Carmo. His lessons inspired me to choose the beautiful field of geometry. I am also grateful to Harold
Rosenberg for the many mathematical discussions and to my students, who provide further motivation in my life. The collaboration and friendship with André has been a constant source of joy to me over the last ten years.

The study of minimal varieties is an old subject that began with the work of Lagrange on the foundations of the calculus of variations. The solution of the Plateau problem for mappings of the disk (Douglas and Rado, 1930) and for rectifiable currents (Federer and Fleming, 1960) are milestones of the field. But the question of existence of closed minimal varieties in general compact Riemannian manifolds is not a problem of minimization. This inspired Almgren (1965) to develop a deep min-max theory for the area functional. His work was improved by his PhD student J. Pitts (1981), but remained largely untouched until the last few years.

André and I were extremely delighted when we discovered that this old theory would play a major role in the solution of the Willmore conjecture. This required a change of perspective: instead of trying to minimize the conformally invariant Willmore functional, as originally proposed, we used conformal transformations to convert the problem into a question of minimizing the maximum of the area of certain five-parameter families of surfaces in the three-sphere. Our work was done mainly while we were both visiting Stanford University at the end of 2011, and the main breakthrough came when we realized how to prove such families are topologically nontrivial. We were very amazed. A few months later we wrote a paper with Ian Agol in which we used similar ideas to solve a conjecture of Freedman, He, and Wang on the Möbius energy of links. Then we turned our attention to the general min-max theory and used it to prove Yau's conjecture about the existence of infinitely many minimal hypersurfaces in the positive Ricci curvature setting. The ideas of Gromov and a paper of Guth on multiparameter sweepouts were very influential. There have been several articles on min-max theory recently, especially by young people, and this makes us very happy. Major questions remain open, such as understanding the index, topology, and multiplicity of these minimal varieties. We hope to contribute further to the field.

\section{Biographical Sketch of Neves}

André Neves is professor of mathematics at Imperial College London. He was born in 1975 in Lisbon, Portugal, completed his $\mathrm{PhD}$ at Stanford University in 2005 under the direction of Richard Schoen, and then held positions at Princeton University from 2005 to 2009, the year he moved to Imperial College London. His field of research is differential geometry, and his work has been distinguished with the New Horizons Prize in Mathematics in 2015, the Royal Society Wolfson Merit Award in 2015, the Whitehead Prize in 2013, and the Leverhulme Prize 
in 2012. He gave the Nirenberg Lectures in 2015 in Montreal, an invited address at the International Congress of Mathematicians in Seoul in 2014, the Rademacher Lectures in 2015 (University of Pennsylvania), and the Barrett Lectures (University of Tennessee) in 2013.

\section{Response from André Neves}

It is a great honor to receive the Oswald Veblen Prize in Geometry along with my dear friend Fernando.

Working and developing min-max theory together with Fernando has been a tremendous experience: it started with an academic interest in conformal deformations of surfaces, but soon we realized that we were discovering some new rich topology in the space of all surfaces. Coupling that with principles of Morse theory and ideas from minimal surfaces theory, we were able to answer some long-standing open questions in geometry. Since its beginnings, variational methods have had great influence in geometry, and I am delighted that our work made some contributions on that front. This is a beautiful subject, and I hope that its contributions will keep increasing for many years to come.

I consider myself very fortunate to have had Richard Schoen-one of the pioneers of geometric analysis-as my $\mathrm{PhD}$ advisor. His mathematical work and sharp intuition have been a towering influence on my research. I would also like to thank my collaborators and friends, from whom I have undoubtedly learned a lot, and my colleague Sir Simon Donaldson for all his support and encouragement throughout my career.

Finally, none of this would have been possible without the constant love and unyielding support of my parents Nelsa and Custódio, my wife Filipa, and our two adorable children, Eva and Tomás. In one way or another, they have all made sacrifices for the pursuit of my career.

\section{About the Prize}

The Oswald Veblen Prize in Geometry is awarded every three years for a notable research memoir in geometry or topology that has appeared during the previous five years in a recognized North American journal (until 2001 the prize was usually awarded every five years). Established in 1961, the prize honors the memory of Oswald Veblen (1880-1960), who served as president of the AMS during 1923-24. The prize was initially established with funds contributed by former students and colleagues and later doubled by Veblen's widow.

In honor of her late father, John L. Synge, who knew and admired Oswald Veblen, Cathleen Synge Morawetz and her husband, Herbert, substantially increased the endowed fund in 2013. Cathleen Synge Morawetz served as president of the AMS during 1995-96. The Prize carries a cash award of US $\$ 5,000$.
The Oswald Veblen Prize in Geometry is awarded by the AMS Council acting on the recommendation of a selection committee. For the 2016 prize, the members of the selection committee were the following individuals.

- Dusa McDuff

- Curtis T. McMullen

- Shmuel Weinberger (Chair)

The complete list of recipients of the Oswald Veblen Prize in Geometry follows.

1964 Christos D. Papakyriakopoulos, Raoul H. Bott

1966 Stephen Smale, Morton Brown, Barry Mazur

1971 Robion C. Kirby, Dennis P. Sullivan

1976 William P. Thurston, James Simons

1981 Mikhael Gromov, Shing-Tung Yau

1986 Michael H. Freedman

1991 Andrew Casson, Clifford H. Taubes

1996 Richard Hamilton, Gang Tian

2001 Jeff Cheeger, Yakov Eliashberg, Michael J. Hopkins

2004 David Gabai

2007 Peter Kronheimer, Tomasz Mrowka, Peter Ozsváth, Zoltán Szabó

2010 Tobias H. Colding, William P. Minicozzi II, Paul Seidel

2013 Ian Agol, Daniel Wise

2016 Fernando Codá-Marques, André Neves 